\title{
Study of the Hot-Spot Temperature during Quench in the Non-Planar Coils of W7-X
}

\author{
Kamil Sedlak, Pierluigi Bruzzone, Thomas Rummel and Michael Nagel
}

\begin{abstract}
The quench protection system of the non-planar coils of the Wendelstein 7-X stellarator was laid out over fifteen years ago. At that time, the assessment of the hot-spot temperature by a basic adiabatic model was done using design values for material and operation parameters. After the operating experience in 2016, the hot-spot temperature is re-assessed with the thermalhydraulic program THEA, using the actual values for delay and dump time. The electrical resistivity of the conduit alloy is measured over the whole range of temperature and in magnetic field on relevant samples of conductor, exposed to the hardening heat treatment after winding. The results are fed into THEA. Parametric variations are studied in the calculations, e.g. testing the effect of the quench initiation zone, delay time and dump resistor. The results suggest that the hot-spot temperature of the conduit in case of quench is safely within the allowable values even in case the dump voltage is reduced compared to the initial design value.
\end{abstract}

Index Terms - hotspot temperature, quench, RRR measurement, W7-X, Wendelstein.

\section{INTRODUCTION}

$\mathbf{T}$ HE WENDELSTEIN 7-X stellarator experienced its first operating campaign in 2016 [1], and now it is a good occasion to think about possible improvements in the operating scenarios and of the machine in general. One of the possible improvement might be related to the operation of the main coils, which are exposed to relatively high voltage $(2.7 \mathrm{kV})$ during the fast current dump, e.g. after a quench detection. The amplitude of the high voltage is related to the decay time constant, which in turn is limited by the maximum allowed hot-spot temperature in the coil. Prolongation of the currentdecay time constant reduces the induced high voltage. At the same time it however increases the hot-spot temperature. As the initial hot-spot temperature assessment was based on a basic adiabatic assessment, and the input parameters to the adiabatic model were based on design material properties, with conservative assumptions in case of uncertainties, we have decided to re-assess the hot-spot temperature using a more sophisticated tool, namely the thermal-hydraulic program THEA [2]. THEA can simulate longitudinal quench propagation (heat propagation along the conductor), and also the transverse heat propagation between different conductor elements (cable, helium, jacket and glass-epoxy insulation). In

K. Sedlak and P. Bruzzone are with the École Polytechnique Fédérale de Lausanne - Swiss Plasma Center, 5232 Villigen PSI, Switzerland (e-mail: kamil.sedlak@psi.ch; pierluigi.bruzzone@psi.ch).

T. Rummel and M. Nagel are with Max-Planck-Institut für Plasmaphysik, 17491 Greifswald, Germany (e-mail: thomas.rummel@ipp.mpg.de; michael.nagel@ipp.mpg.de).

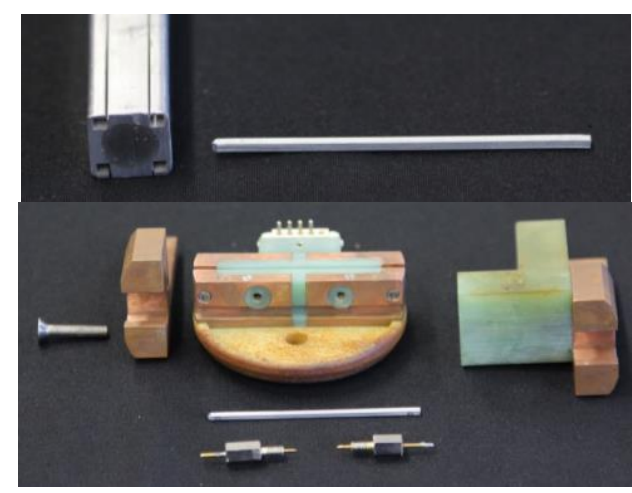

Fig. 1. Upper picture: Sample (thin bar on the right) of jacket alloy cut out by wire erosion from a short conductor length (left). Lower picture: sample holder used in the resistivity measurement. The sample is inserted in the holder, and the voltage is read out by two pins (at the bottom of the photograph) inserted into two holes of the sample holder. The distance between the two voltage taps is $30 \mathrm{~mm}$.

addition, the resistive voltage over the full conductor length can be calculated, and consequently a realistic quench detection based on exceeding a voltage threshold value as in the real coil operation can be simulated. The operating parameters and material data are now known with a higher confidence, and the hot-spot temperature assessment can therefore be done more reliably and without unnecessary conservatism. The results of the study may in the end lead to a safer coil operation.

\section{REsistivity MEASUREMENT OF THE CONDUit Alloy}

One of the important inputs in the hot-spot calculation is the resistivity of conductor conduit made of an aluminium-based alloy. As the non-planar coil required conductor bending with the bending radius as small as $120 \mathrm{~mm}$, the conduit alloy had to be soft enough during the coil production, and stable enough to carry the loads during operation. The aluminum alloy $\mathrm{AlMgSi} 0.5$ was chosen [3], [4], and the required yield strength was achieved during an annealing hardening process after the coil winding. A short conductor length, a left-over piece of conductor of a non-planar coil AAB51, was available at IPP. The conductor piece experienced the whole coilmanufacturing process including the hardening, which likely influences the resistivity of the jacket alloy.

The cryogenic resistivity measurement of the conduit alloy of W7-X conductor was performed at the Swiss Plasma Center (SPC). Four samples were cut by wire erosion out of the short conductor length. The sample cross section, typically $1.98 \times 1.98 \mathrm{~mm}$, was accurately measured at three different locations for all four samples. The average of the three meas- 
TABLE I

RESISTIVITY OF FOUR SAMPLES MEASURED IN FEBRUARY 2017 AT SPC at Zero Magnetic FIELD

\begin{tabular}{cccccccc}
\hline \hline \multicolumn{2}{c}{ Sample 1 } & \multicolumn{2}{c}{ Sample 2 } & \multicolumn{2}{c}{ Sample 3 } & \multicolumn{2}{c}{ Sample 4 } \\
Temp. & $\rho$ & Temp. & $\rho$ & Temp. & $\rho$ & Temp. & $\rho$ \\
$\mathrm{K}$ & $\mathrm{n} \Omega \cdot \mathrm{m}$ & $\mathrm{K}$ & $\mathrm{n} \Omega \cdot \mathrm{m}$ & $\mathrm{K}$ & $\mathrm{n} \Omega \cdot \mathrm{m}$ & $\mathrm{K}$ & $\mathrm{n} \Omega \cdot \mathrm{m}$ \\
\hline 4.2 & 8.2 & 4.2 & 8.2 & 4.2 & 8.2 & 4.2 & 8.3 \\
20.3 & 8.2 & 19.9 & 8.3 & 21.0 & 8.3 & 19.9 & 8.3 \\
39.5 & 8.6 & 41.3 & 8.8 & 38.0 & 8.7 & 39.0 & 8.7 \\
77 & 11.0 & 77 & 11.1 & 77 & 11.0 & 77 & 11.1 \\
295 & 36.8 & 290 & 35.7 & 293 & 35.8 & 291 & 35.5 \\
\hline \hline
\end{tabular}

urements was used in the assessment. An illustration of the sample and sample holder is shown in Fig. 1.

The resistance was measured at five different temperatures between $4.2 \mathrm{~K}$ and $300 \mathrm{~K}$. The measured data are provided in Table I and Fig. 2. We estimate the measurement accuracy at $3 \%$. The dominant source of uncertainty originates from the assessment of the voltage-current curves plotted on paper by the measuring device Yokogawa 3023. The other sources of error, namely the uncertainty on the distance of voltage taps, uncertainty on the cross-section measurement of the samples, and accuracy of temperature measurement are only minor contributions to the overall measurement error.

The measured resistivity was fitted by the function $\rho=8.20+\frac{13.7 \cdot T^{2.4}}{1+15.64 \cdot T^{1.71} \cdot \exp (134.1 / T)} \quad[\mathrm{n} \Omega \cdot \mathrm{m}]$

where $T$ is the temperature in $\mathrm{K}$. The RRR calculated from the fit is $R R R=\rho_{273 \mathrm{~K}} / \rho_{4.2 \mathrm{~K}}=4.14$.

Also the magnetoresistance at $4.2 \mathrm{~K}$ at magnetic field of $3 \mathrm{~T}$ and $5 \mathrm{~T}$ was measured. The increase of resistance with magnetic field is very weak. The relative increase of resistivity with magnetic field can be expressed as

$$
R(B, 4.2 \mathrm{~K})=R(0 \mathrm{~T}, 4.2 \mathrm{~K}) \cdot(1+0.0048 \cdot B),
$$

where magnetic field $\mathrm{B}$ is in $\mathrm{T}$.

\section{Simulation MODEL}

The quench calculations presented in this paper are based on program THEA from Cryosoft [2]. The model of the W7-X non-planar coil consists of three thermal elements, namely

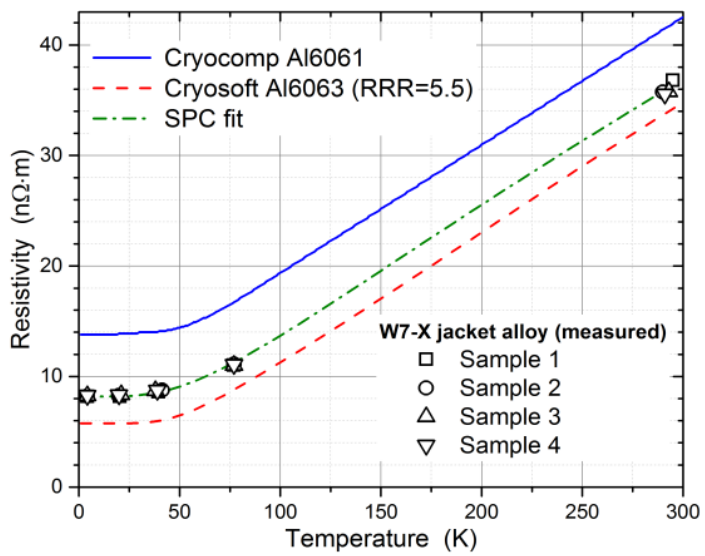

Fig. 2. Resistivity measurement of the W7-X jacket alloy, including the fit of eq. (1), compared with the parameterizations of resistivity of aluminium alloys implemented in Cryocomp (Al6061-T6) [5] and Cryosoft (A16063) [1] programs, which are supposed to be most similar to the actual jacket alloy (from the alloys implemented in the mentioned programs).
TABLE II

FOUR DIFFERENT SCENARIOS USED AS INITIAL CONDITIONS IN THE QUENCH SiMULATIONS

\begin{tabular}{ccccc}
\hline \hline & Scenario 1 & Scenario 2 & Scenario 3 & Scenario 4 \\
\hline Current, kA & 18.2 & 18.2 & 18.2 & 15.32 \\
$B, \mathrm{~T}$ & 6.7 & 6.7 & 6.7 & 5.64 \\
$T$ inlet, $\mathrm{K}$ & 3.80 & 3.40 & 4.40 & 4.10 \\
$T$ outlet, $\mathrm{K}$ & 3.99 & 3.64 & 4.51 & 4.14 \\
$p$ inlet, bar & 4.45 & 4.45 & 4.45 & 3.62 \\
$p$ outlet, bar & 3.14 & 3.14 & 3.14 & 3.26 \\
$\Delta p, \mathrm{bar}$ & 1.31 & 1.31 & 1.31 & 0.36 \\
$d m / d t$ of $\mathrm{He}, \mathrm{g} / \mathrm{s}$ & 1.23 & 1.24 & 1.20 & 0.59 \\
\hline \hline
\end{tabular}

$\mathrm{NbTi}$ strands, aluminium-based alloy jacket and glass-epoxy insulation wrap; and one hydraulic element, helium. The heat exchange coefficient between helium and strands and between helium and aluminium jacket is defined as maximum of smooth-tube and Dittus-Boelter correlation. Expressed in Nusselt number, it reads: $\mathrm{Nu}=\operatorname{Max}\left(4.0,0.023 \cdot \mathrm{Re}^{0.8} \cdot \mathrm{Pr}^{0.4}\right)$, where Re is the Reynolds number, and Pr the Prandtl number.

The heat exchange coefficient between strands and $\mathrm{Al}$ jacket is assumed to be $500 \mathrm{~W} /\left(\mathrm{m}^{2} \mathrm{~K}\right)$ with the touching perimeter equal to $10 \%$ of the cable circumference. The numerical value of $500 \mathrm{~W} /\left(\mathrm{m}^{2} \mathrm{~K}\right)$ is typically used in absence of a better knowledge of the heat exchange coefficient [6]. Also the heat exchange coefficient between $\mathrm{Al}$ jacket and insulation layer is supposed to be $500 \mathrm{~W} /\left(\mathrm{m}^{2} \mathrm{~K}\right)$, where the touching perimeter is the outer perimeter of $\mathrm{Al}$ jacket.

The considered friction coefficient of the He flow is DarcyForcheimer-Bagnasco-Bottura-Lewandowska equation for porous media [7]. Heat conduction between neighboring turns and layers is not accounted.

Two operating current cases will be investigated in following sections. The first one, $18.2 \mathrm{kA}$ case, corresponds to 2016 operation with a pure nickel resistor $\left(148 \mathrm{~m} \Omega\right.$ at $\left.20^{\circ} \mathrm{C}\right)$ with relatively fast current dump. Since the dump resistance is temperature dependent, the current decay is not exponential (the current decay curve is shown in Fig. 3). The current drops to its half value (from $18.2 \mathrm{kA}$ to $9.1 \mathrm{kA}$ ) in $1.9 \mathrm{~s}$, and the maximum voltage goes up to $2.7 \mathrm{kV}$. In the second case, the operating current is lowered to $15.32 \mathrm{kA}$, and the dump resistor is modified (internally denoted variant 2 in [8] with $74 \mathrm{~m} \Omega$ at $20^{\circ} \mathrm{C}$ ). The corresponding current decay is significantly slower, half value $(15.32 \mathrm{kA}$ to $7.66 \mathrm{kA})$ is reached in $3.6 \mathrm{~s}$, and the maximum voltage is reduced to $1.8 \mathrm{kV}$. In both cases, the magnetic field is considered to be constant along the conductor length, and equal to $6.7 \mathrm{~T}$ and $5.64 \mathrm{~T}$, respectively.

Before simulating the quench, a steady-state calculation is performed. Four different steady-state runs were simulated, see Table II. The conductor temperature in scenario 1 is considered to be the most relevant for the non-planar coil operation at $18.2 \mathrm{kA}$. The scenarios 2 and 3 are two extreme cases, the former with very low, the latter with high conductor temperature for the $18.2 \mathrm{kA}$ operating current. Finally, scenario 4 is considered to be the most relevant for operating the coil at $15.32 \mathrm{kA}$. Once the steady state is reached, the mass flow rate and temperature and pressure profile along the conductor length is obtained for specified boundary conditions, and later 
on used as the initial conditions of the quench simulations.

The assumed conductor length was $146 \mathrm{~m}$. The temperature slowly increases over the conductor length as a consequence of Joule-Thomson effect, while pressure decreases linearly between the inlet and outlet.

\section{QUENCH SimULATION}

\section{A. Nominal Simulation at $18.2 \mathrm{kA}$}

In the nominal quench assessment, the quench is initiated by heating $10 \mathrm{~cm}$ long cable zone at the location $80.0 \mathrm{~m}$ away from the conductor inlet, close to the center of a layer. The initial temperature and pressure distribution along the conductor length correspond to scenario 1 . The quench initiating energy is $2 \mathrm{~J}$, which is deposited in form of heat to the NbTi strands within initial $0.1 \mathrm{~s}$ of the simulation. The minimum quench energy in the simulation (found iteratively in THEA) at these particular conditions and at initial conditions of scenario 1 is $1.38 \pm 0.03 \mathrm{~J}$.

The overall resistive voltage, measured over the full conductor length, rises during and after the initial heating. $0.151 \mathrm{~s}$ after the heating is initiated, the voltage reaches the specified detection threshold of $0.15 \mathrm{~V}$, and the quench is "detected". At this moment, the highest $\mathrm{NbTi}$ temperature in the normal zone is around $26 \mathrm{~K}$. The current is still kept at the maximum of $18.2 \mathrm{kA}$ for additional $350 \mathrm{~ms}(50 \mathrm{~ms}$ of the validation time plus $300 \mathrm{~ms}$ for switching time of the breakers). At the time $0.501 \mathrm{~s}$, current starts to decay according to the decay function calculated by IPP [8] for the original discharge resistor (see the current line in Fig. 3).

Resistivity of the jacket alloy measured at SPC and parametrized in eq. (1) is used in the nominal quench assessment. The temperature evolution during quench is shown in Fig. 3. The maximum temperature, i.e. the hot-spot temperature, observed during the quench is $99 \mathrm{~K}$ in the NbTi cable and $98.1 \mathrm{~K}$ in the jacket.

Quench evolution longitudinally along the conductor length is presented in Fig. 4. There is a clear peak around the position of $\mathrm{x}=80 \mathrm{~m}$, where the quench was initiated. The quench propagates very quickly with the speed of $\sim 100 \mathrm{~m} / \mathrm{s}$ in the longitudinal direction. In the absence of a dedicated pressurerelief cooling channel the pressure build-up to relatively high values is unavoidable.

\section{B. Parametric studies at $18.2 \mathrm{kA}$}

There were several uncertainties in the assessment of the hot-spot temperature in the previous section. We therefore varied several parameters in order to study the sensitivity of the predicted hot-spot temperature on the assumptions in our assessment.

First of all, we changed the initial conditions in the coil, namely the initial temperature in the steady state just before the quench is initiated. Three different scenarios specified in Table II were used. Almost no difference in hot-spot temperature is observed between the nominal scenario 1 , where the initial temperature at the quench initiation zone is $3.91 \mathrm{~K}$, and scenario 2 with initial temperature of $3.54 \mathrm{~K}$. In the latter case,

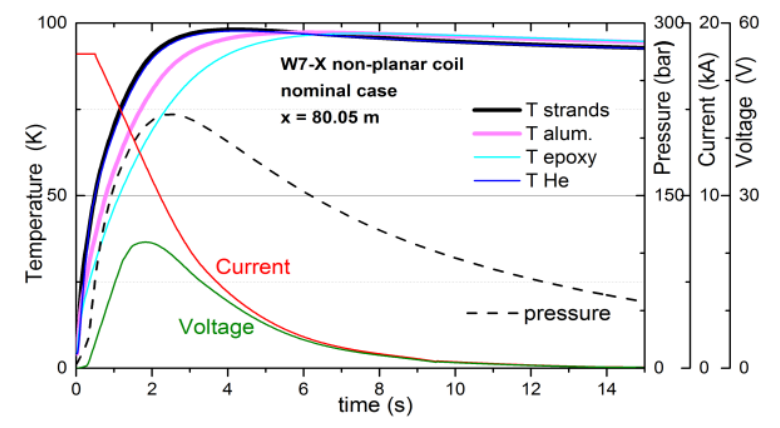

Fig. 3. Quench evolution at position $x=80.05 \mathrm{~m}$, where the quench was initiated. The voltage is only the resistive one, no inductive voltage is considered in the calculation.

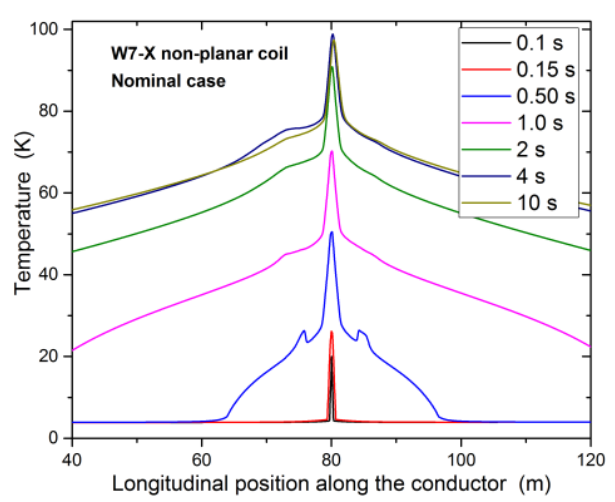

Fig. 4. Strand temperature evolution along the conductor longitudinal position.

the hot-spot temperature in the jacket is $99.7 \mathrm{~K}$, only $1.6 \mathrm{~K}$ higher than in scenario 1 . The situation is slightly different for scenario 3 with initial temperature of $4.47 \mathrm{~K}$, in which case the jacket hot-spot temperature is only $92.7 \mathrm{~K}$. The temperature margin of the superconductor in scenario 3 is small, and the quench, once provoked by heating, can propagate longitudinally faster compared to the scenarios with a lower initial temperature. Consequently, the initial normal zone at the moment when the voltage exceeds the threshold of $0.15 \mathrm{~V}$ is broader, with less pronounced peak in temperature. The peak, visible also in Fig. 4, becomes less pronounced over the whole duration of the quench, leading to a lower hot-spot temperature.

An important effect on the hot-spot temperature has the electric resistivity of the jacket. Assuming resistivity temperature dependence of Cryosoft Al6063 (full line in Fig. 2) or Cryocomp Al6061-T6 (dashed line in Fig. 2) leads to the jacket hot-spot temperature of $90.0 \mathrm{~K}$ and $111.1 \mathrm{~K}$, respectively. The measurement of jacket alloy resistivity therefore significantly reduced the uncertainty of the hot-spot temperature assessment.

There is some uncertainty about the thermal resistance between different thermal elements, especially on the boundary between the jacket and glass epoxy insulation. In order to see the effect of the heat transfer to the glass epoxy layer, we increased the thermal resistance between jacket and insulation to infinity. This effectively discards the insulation from the assessment. Doing so, the hot-spot temperature increased from 98.1 $\mathrm{K}$ to $103.7 \mathrm{~K}$. We can conclude that the heat transfer to the glass-epoxy slightly reduces the hot-spot temperature, but 


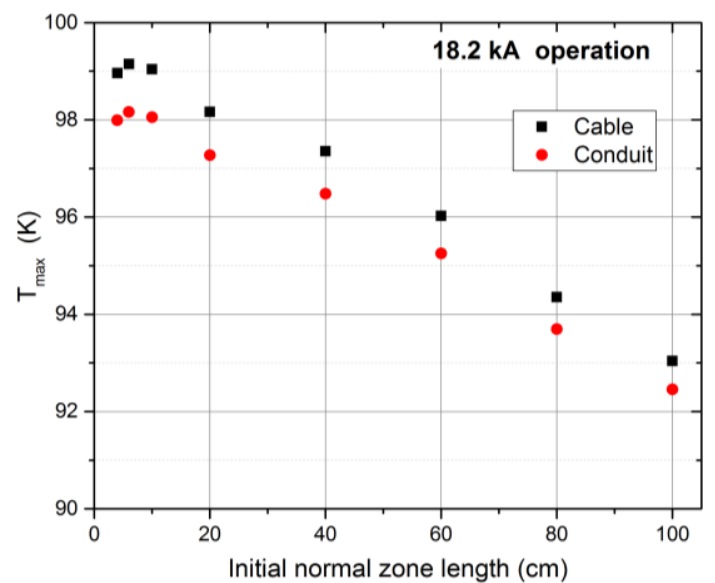

Fig. 5. Hot-spot temperature as a function of initial normal zone length.

the effect is relatively small.

If also the heat transfer between the cable and conduit is blocked, i.e. both strand-conduit and helium-conduit thermal resistances are set to infinity, all ohmic heat generated in the $\mathrm{NbTi}$ strands is deposited in the strands and helium only. The temperature of the jacket nevertheless increases due to the ohmic self-heating by the current transferred through the conduit during the quench, as THEA appropriately distributes the current transferred through the conductor among the thermal elements, depending on the temperature-dependent resistance of the respective element. The conduit temperature, $80.1 \mathrm{~K}$, significantly differs from the temperature of the cable, $113.5 \mathrm{~K}$ ), but the effect of jacket self-heating is anyway significant. The main conclusion of this unrealistic case is that even if the assumed heat transfer coefficients between strands, helium and conduit were not perfect, the error on the assessed hotspot temperature would not be dramatic.

In the simulation, as in reality, there is some delay time $t_{\mathrm{d}}$, during which current remains at its full value after voltage reaches the threshold of $0.15 \mathrm{~V}$. The delay time consists of $50 \mathrm{~ms}$ validation time plus some "switching" time for opening of breakers. In nominal case, the switching time is $300 \mathrm{~ms}$, and the total delay time is therefore $350 \mathrm{~ms}$. As expected, the hot-spot temperature rises with increasing delay time (not shown), however the increase is not dramatic in a reasonable experimental range of delay times. The maximum conduit temperature is $96 \mathrm{~K}$ for $t_{\mathrm{d}}=300 \mathrm{~ms}$, and $100 \mathrm{~K}$ for $t_{\mathrm{d}}=400 \mathrm{~ms}$.

The effect of the length of the initial normal zone, i.e. the cable length where the quench initiation energy is deposited, is shown in Fig. 5. Always the same energy per conductor length is deposited, namely $20 \mathrm{~J} / \mathrm{m}$. The hot-spot temperature slightly decreases with the length of the quench initiation zone, and our nominal case that uses $10 \mathrm{~cm}$ long initiation zone is a conservative choice around the maximum of the curve.

A sensitivity test of the hot-spot calculation on the quench initiation energy is studied in Fig. 6. The heat energy in the range between $1.38 \mathrm{~J}$ and $100 \mathrm{~J}$ was deposited in the NbTi cable, in the quench initiation zone of $10 \mathrm{~cm}$ over a time of $0.1 \mathrm{~s}$. Ideally, the hot-spot temperature should not depend on the quench initiation energy, a kind of a technical parameter. This is in fact the case for at least one order of magnitude of the

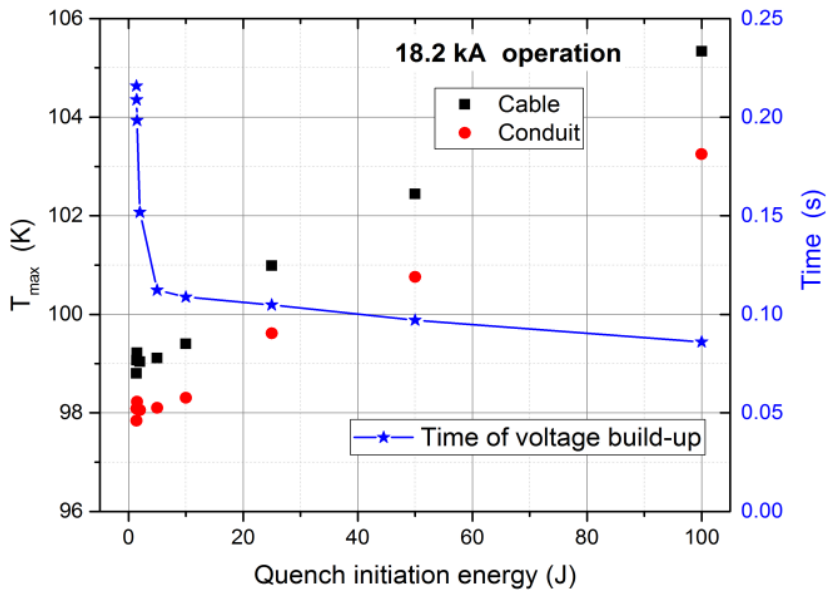

Fig. 6. Hot-spot temperature as a function of quench initiation energy, i.e. the energy that heats up the initial $10 \mathrm{~cm}$ long region of the cable during the time of $0.1 \mathrm{~s}$, and induces the quench.

quench initiation energies between the minimum quench energy, MQE (1.38 J) and 10.MQE (13.8 J).

In order to check the effect of boundary conditions (pressure and temperature at conductor edges), we performed a simulation with closed conductor inlet and outlet, with initially stagnant helium. When conductor ends were completely closed, the hot-spot temperature was $3 \mathrm{~K}$ higher compared to the "reservoir" boundary conditions (constant pressure).

\section{Hot-spot temperature for $15.32 \mathrm{kA}$ operation}

The calculated hot-spot temperature of $98 \mathrm{~K}$ (jacket) for the coil operation at $18.2 \mathrm{kA}$ is significantly below the criterion internally set by IPP to $130 \mathrm{~K}$. The available margin in the hot-spot temperature could be exploited in reduction of the dump voltage by slowing down the current during the fast current dump.

In order to investigate this option, a slower current decay function, corresponding to a modified dump resistor, was calculated by IPP. The corresponding hot-spot assessment based on initial conditions of scenario 1 leads to the maximum temperature of $156 \mathrm{~K}$, exceeding the acceptable hot-spot limit.

The situation changes when also the operating current is reduced from $18.2 \mathrm{kA}$ to $15.32 \mathrm{kA}$, corresponding to the next lower possible W7-X non-planar coil operating current. The hot-spot temperature with slower current decay is only $116 \mathrm{~K}$, both in the cable and jacket. In this case the initial conditions correspond to scenario 4 (Table II) adjusted for $15.32 \mathrm{kA}$ operation, with less powerful cooling compared to scenarios 1-3 that is necessary for $18.2 \mathrm{kA}$ operation.

\section{CONCLUSIONS}

The results indicate that the maximum temperature (hotspot temperature) calculated by THEA in the investigated nominal $18.2 \mathrm{kA}$ scenario $\left(\mathrm{B}=6.7 \mathrm{~T}, \mathrm{I}_{\mathrm{op}}=18.2 \mathrm{kA}, \mathrm{t}_{\text {de- }}\right.$ lay $=350 \mathrm{~ms})$ is $99 \mathrm{~K}$, and in the $15.32 \mathrm{kA}$ scenario $\left(\mathrm{B}=5.64 \mathrm{~T}, \mathrm{I}_{\mathrm{op}}=15.32 \mathrm{kA}, \mathrm{t}_{\text {delay }}=350 \mathrm{~ms}\right.$, slower current decay) it is $116 \mathrm{~K}$.

An important influence on the hot-spot temperature comes from resistance (RRR) of the aluminium alloy of the jacket. 
As the jacket resistivity was measured at SPC, this big source of uncertainty has been eliminated. The remaining uncertainties in the hot-spot temperature assessment are quite limited, within a few Kelvin. As the transverse heat conduction between neighboring turns and layers was not considered, the hot-spot assessment remains conservative.

\section{REFERENCES}

[1] R. C. Wolf et al., "Major results from the first plasma campaign of the Wendelstein 7-X stellarator", Nucl. Fusion 57, 2017, Art. no. 102020.

[2] THEA software, Cryosoft: http://botturl.home.cern.ch/botturl/CryoSoft/.

[3] T. Rummel et al., "The Superconducting Magnet System of the Stellarator Wendelstein 7-X", IEEE Trans. Appl. Supercond., vol. 40, p. 769776, 2012.

[4] H. Ehmler, J. Baldzuhn, L. Genini, K. Heyn, C. Sborchia, T. Schild, W7-X Team, "Review of the acceptance tests of the W7-X superconducting magnets," Fusion Engineering and Design 82, p. 1406-1412, 2007.

[5] Cryocomp Software. Available: http://www.eckelsengineering.com/.

[6] L. Savoldi, R. Zanino, "Common approach for thermal-hydraulic calculations", Eurofusion internal report, EFDA_D_2LMECE, 2016.

[7] M. Bagnasco, L. Bottura and M. Lewandowska, "Friction factor correlation for CICC's based on a porous media analogy," Cryogenics, vol. 50, p. 711-719, 2010.

[8] T. Rummel, "Collection of the W7-X superconductor data to be used for the optimization of the discharge resistors", version 1-AAE-T0023.0 (30.1.2017). Available on request. 